\title{
La alimentación de Munida gregaria (Fabricius, 1793) (Crustacea: Anomura: Galatheidae) en fondos de pesca del Golfo San Jorge, Argentina
}

\author{
Diet of Munida gregaria (Fabricius, 1793) (Crustacea: Anomura: Galatheidae) \\ in fishing beds of the San Jorge Gulf, Argentina \\ Martín Varisco ${ }^{1}$ y Julio H. Vinuesa ${ }^{2}$ \\ ${ }^{1}$ Facultad de Ciencias Naturales, Universidad Nacional de la Patagonia San Juan Bosco. Campus Universitario. Ruta 1 Km 4 (9000) \\ Comodoro Rivadavia, Chubut, Argentina \\ ${ }^{2}$ Centro de Desarrollo Costero. Facultad de Humanidades y Ciencias Sociales, U.N.P.S.J.B y CONICET. Campus Universitario. \\ Ruta 1 Km 4 (9000) Comodoro Rivadavia, Chubut, Argentina \\ martinvarisco@hotmail.com
}

\begin{abstract}
The area encompassing San Jorge gulf and surroundings is one of the most important fishing grounds in Argentina. Heavy fishing pressure affects the dynamics of natural communities. The discards of low commercial value species may have an impact on benthic-demersal communities by increasing the amount of organics in the seafloor. This accumulation initially favours generalist species, which are capable of ingesting the available organic matter; such is the case of the lobster krill Munida gregaria. This study evaluates if the lobster krill benefits from discards of the different fisheries in the gulf and how the species adjusts to local conditions caused by the fishing activity. In order to achieve these goals, five bottom samples were taken from the central region and five from the north of the San Jorge gulf. The dietary composition of 30 specimens from each sample was determined, and statistical analyses were performed to test for differences among sampling sites. The results recognized significant differences among sampling sites in the diet of the lobster krill, the larger consumption of decapod crustaceans in the northern section of the gulf was attributed to its larger abundance in the bycatch composition.
\end{abstract}

Key words: Feeding strategy, lobster krill, fishery, bycatch, discard

\section{Introducción}

La langostilla Munida gregaria (Fabricius, 1793) es una especie polimórfica dentro en la amplia área de distribución circumsubantártica. Williams (1973, 1980) demostró en Australia, que las especies mencionadas
Resumen.- El Golfo San Jorge y sus inmediaciones concentran una parte importante de la actividad pesquera de la Argentina. Esta actividad antrópica, cuando es intensa, afecta la dinámica natural de las comunidades sobre las que se desarrolla. El descarte de especies de menor rentabilidad es una forma importante de impacto sobre las comunidades bentónico-demersales, pues aporta elevadas concentraciones de materia orgánica sobre los fondos. Esta acumulación favorece inicialmente a las especies generalistas, capaces de incorporar la materia orgánica disponible, entre ellas la langostilla Munida gregaria. Este estudio buscó demostrar si la especie capitaliza el descarte generado por las distintas pesquerías del golfo y como se adecua a condiciones locales dadas por la actividad pesquera. Para ello se tomaron cinco muestras provenientes del sector central y otras cinco en los fondos de pesca del norte del Golfo San Jorge. Se analizó la composición de la dieta de 30 ejemplares por muestra y se realizaron pruebas estadísticas a fin de establecer diferencias entre los sectores muestreados. Las mismas mostraron que la dieta es sensiblemente diferente entre estos sitios, y que dichas diferencias se deben principalmente a la elevada ingesta de crustáceos decápodos en el área norte del golfo, donde hay mayor presencia de los mismos en los descartes.

Palabras clave: Estrategia de alimentación, langostilla patagónica, pesquerías, captura incidental, descarte

como M. gregaria y M. subrugosa, correspondían a dos morfotipos de la especie $M$. gregaria. Basados en diferencias morfológicas y de nicho ecológico ambos morfotipos fueron considerados especies válidas (Boschi et al. 1992, Rodríguez \& Bahamonde 1986, Lovrich et al. 1998). Estudios recientes de distancias 
genéticas y cruzamientos aportan nuevas pruebas sobre la identidad de estos morfotipos y apoyan la existencia de una única especie (Pérez-Barros et al. 2005, PérezBarros et al. en prensa). El primero tiene hábitos pelágicos durante la primera parte de su vida y el segundo es epibentónico en su fase juvenil y adulta. La otra especie de la familia Galatheidae citada para el mar argentino es Munida spinosa Henderson, 1885, frecuente en profundidades mayores de $100 \mathrm{~m}$ (Boschi et al. 1992) y también hallada en el sector externo del golfo (Roux et al. 1995). M. gregaria es la única especie de galateido citada para el Golfo San Jorge, sitio en el que sólo se ha hallado el morfotipo "subrugosa" (Vinuesa 2005).

Los galateidos son especies claves en las comunidades debido principalmente a que ocurren en densidades altas y hacen más efectivo el flujo de energía en las mismas, siendo un nexo entre los niveles basales y los predadores tope (Romero et al. 2004). Dado su potencial económico e importancia trófica, es amplia la información sobre la biología de los galateidos en todo el mundo (Rodríguez \& Bahamonde 1986, Pérez Flores \& Aurioles Gamboa 1995, Zeldis 1985). Las poblaciones de $M$. gregaria del canal Beagle han sido ampliamente estudiadas, conociéndose datos sobre su reproducción (Tapella et al. 2002), crecimiento, distribución, abundancia (Tapella 2002) y posición trófica (Romero et al. 2004). Es menor la información biológica de las poblaciones del Golfo San Jorge, sitio donde la especie desarrolla un papel clave en la comunidad bentónico demersal, en la que se incluyen importantes recursos pesqueros. La especie se alimenta de depósitos superficiales al tiempo que es presa de predadores tope (Vinuesa \& Varisco no publicado). En este golfo, el periodo reproductivo se inicia en julio y se prolonga hasta diciembre cuando se producen los reclutamientos (Vinuesa 2007). $M$. gregaria es una especie característica de las comunidades de fondos blandos, y junto al cangrejo araña, Eurypodius latreillei, son los decápodos mas importantes de la comunidad bentónica del golfo (Roux 2000).

La langostilla es similar en tamaño y quizás en abundancia a otras especies que se explotan con una rentabilidad aceptable; tal es el caso de los langostinos Cervimunida johni (Porter, 1903) y Pleuroncodes monodon (H. Milne Edwards, 1937) de Chile (Lovrich et al. 1998), por lo que representa un potencial recurso pesquero para la cuenca del Golfo San Jorge, Argentina. La especie es uno de los componentes más importantes de la captura acompañante en las pesquerías del golfo. En 2004, la pesca de langostino patagónico Pleoticus muelleri (Bate, 1888) ocasionó un descarte declarado cercano a las tres mil toneladas de langostilla, además de miles de toneladas de otras especies ${ }^{1}$.

La captura incidental de especies y el posterior descarte de las mismas conlleva distintos tipos de cambios en las comunidades bentónico-demersales y numerosos trabajos realizados en distintas partes del mundo aportan datos al respecto (Goñi 1998, Kaiser \& Spencer 1995, Goñi 1998, Philippart 1998, Stobutzki et al. 2001, Davis 2002, Gislason 2003, entre otros). En el área central del Golfo San Jorge se han mencionado fondos anóxicos cubiertos de restos de crustáceos, con peces y elevados porcentajes de lesiones y pérdida de apéndices en centollas (Vinuesa et al. 1998). Otros estudios realizados en el área de pesca del langostino, han señalado la inexistencia de efectos pronunciados sobre fondos blandos, dado que no se han observado cambios en la composición de la comunidad bentónica (Roux \& Fernández 1997, Roux 2000).

Dado que $M$. gregaria concentra sus hábitos alimenticios principalmente en la alimentación de depósitos superficiales de materia orgánica, macroinfauna y la carroña (Vinuesa \& Varisco, no publicado), el descarte pesquero influiría en la composición de la dieta de la especie. El presente trabajo tiene como objetivo evaluar la influencia de la actividad pesquera en la alimentación de $M$. gregaria, comparando para ello la dieta de la especie en zonas donde la actividad pesquera presenta características diferentes.

\section{Material y métodos}

Las muestras fueron recolectadas por barcos pesqueros costeros y fresqueros que operan desde los puertos de Comodoro Rivadavia y Caleta Córdova, en Chubut y Caleta Paula en Santa Cruz, Argentina. Las muestras fueron conservadas en alcohol etílico $70 \%$ mientras permanecieron a bordo para asegurar una fijación rápida, y luego fueron congeladas hasta su utilización en el laboratorio.

Se tomaron diez muestras en lances realizados con red de arrastre de fondo, cinco de la cuales corresponden

\footnotetext{
${ }^{1}$ CEDEPESCA

http://www.cedepesca.org.ar/noticias/descartes_2004.html
} 
a los fondos de pesca de langostino en el norte del Golfo San Jorge, mientras que las restantes fueron tomadas en el área central del golfo (Fig. 1).

En el área norte del golfo operan buques fresqueros y congeladores, siendo el langostino patagónico $P$. muelleri la especie blanco durante gran parte del año. En el área central frente a las ciudades de Comodoro
Rivadavia y Caleta Olivia, la pesquería se lleva a cabo por buques de rada-ría y también fresqueros, los cuales desarrollan una actividad de captura multiespecífica (Bertolotti et al. 2001), donde se incluyen la merluza común Merluccius hubbsi, abadejo Genypterus blacodes, pez gallo Callorhinchus callorhynchus, centolla Lithodes santolla, etc.

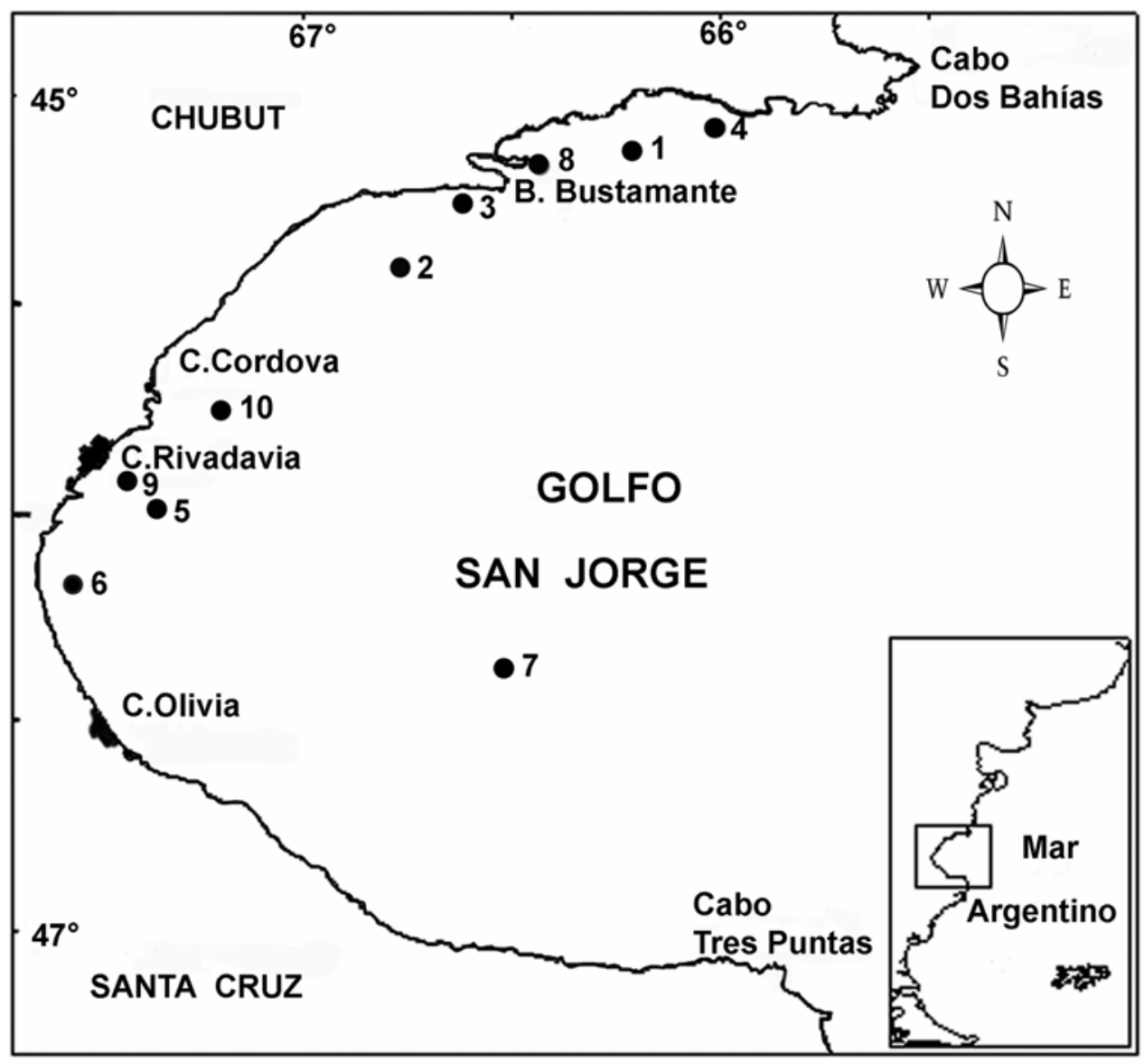

Figura 1

Áreas de muestreo en el Golfo San Jorge, Argentina, en el Océano Atlántico sur occidental. Los puntos muestran la localización de las capturas. Las muestras M1, M2, M3, M4 y M8 corresponden al sector norte. Las muestras M5, M6, M7, M9 y M10 corresponden al área central del golfo

Sampling areas in the San Jorge Gulf, Argentina, in the southwestern Atlantic Ocean. The dots show the location of the catches. Samples M1, M2, M3, M4 and M8 belong to the northern area. Samples M5, M6, M7, M9 and M10 belong to the central area of the gulf 
Se estudió un total de 300 individuos adultos de ambos sexos. En todos ellos se procedió a la identificación del sexo y se midió la longitud del caparazón (LC), que es la distancia entre el margen orbital posterior hasta el margen posterior medio del caparazón (Romero 2003). Luego se extrajeron los estómagos y su contenido fue separado cuidadosamente del órgano. Se observaron inicialmente bajo microscopio estereoscópico con 20 aumentos, con el objeto de identificar los componentes de mayor tamaño y luego se los colocó en tubos Eppendorf fijados con alcohol 70\%. Se homogeneizó la muestra y se extrajo un mililitro del contenido estomacal, el cual fue observado minuciosamente bajo microscopio óptico con una magnificación de 100 aumentos, para establecer la importancia de los diferentes componentes de la dieta. Se estimaron la frecuencia de ocurrencia (FO) y la abundancia relativa (AR) de los ítems hallados. Los mismos fueron determinados al menor nivel taxonómico posible.

La FO de cada ítem alimentario se calculó como $\mathrm{FO}=\mathrm{Ni} \times \mathrm{N} \mathrm{t}^{-1} \times 100$, donde $\mathrm{Ni}$ es el número de estómagos con el ítem i y $\mathrm{Nt}$ es el número total de estómagos de una determinada muestra. La AR resalta la importancia de cada ítem alimentario en la dieta del animal. El valor de AR se calculó como $A R=\mathrm{i}_{\mathrm{a}} \times \sum_{\mathrm{i}}^{-1}$ $\mathrm{x} 100$, donde $\mathrm{i}_{\mathrm{a}}$ es el número de veces que aparece un determinado ítem, y la sumatoria corresponde al total (Romero et al. 2004).

Los valores de FO y AR obtenidos fueron analizados mediante el método de análisis de similitudes (ANOSIM) para determinar las posibles diferencias entre el área norte $\mathrm{y}$ el área central. Posteriormente los valores de AR fueron usados para la construcción de una matriz de similitud, utilizando el método de escalamiento multidimensional (MDS) que permite el agrupamiento de las muestras (Penchaszadeh et al. 2004). También se realizó un análisis de porcentajes de similitud (SIMPER) para cuantificar la contribución de los grupos en la diferencia entre los sitios (Clarke \& Warwick 2001).

\section{Resultados}

La talla de los machos varió entre 16,4 y $28,1 \mathrm{~mm}$ y la de las hembras, entre 13,1 y $22 \mathrm{~mm}$ de LC. La proporción de machos y hembras fue de 2,4:1. Las muestras se caracterizaron por la presencia elevada de sedimentos en los contenidos estomacales, la ausencia casi total de macroalgas $y$ un grado alto de descomposición de los ítems alimenticios, lo cual limitó el alcance de la determinación taxonómica. Unos pocos grupos taxonómicos están involucrados en la alimentación, en todas las muestras poliquetos, crustáceos decápodos y MOP constituyen más del 50\% de la dieta. Algunos grupos de protistas entre los que encontramos foraminíferos, diatomeas $y$ en menor medida dinoflagelados, están pobremente representados en términos de abundancia relativa pero presentan valores altos de ocurrencia (Tablas 1 y 2 ).

Los restos celulares semidigeridos no identificables fueron agrupados en la categoría "indeterminados", mientras que en las abundancias relativas la categoría "otros" incluye componentes cuyo valores son bajos; tal es el caso de restos de peces, equinodermos, poríferos y crustáceos inferiores como copépodos, anfípodos y ostrácodos.

La materia orgánica particulada (MOP) está constituida por agregados amorfos de materia orgánica en descomposición, acomplejada con microorganismos. Este fue el componente más frecuente y abundante en la dieta de la especie en todas las muestras analizadas (Tablas 1 y 2 ). Entre los crustáceos decápodos identificados se encuentran el langostino patagónico $P$. muelleri y también la langostilla $M$. gregaria, constituyendo los componentes más abundantes, luego de la MOP.

Se encontraron diferencias altamente significativas entre los sitios norte y centro respecto de la FO $(P=$ $0,008)$ y también respecto de la $\operatorname{AR}(P=0,007)$. En el área central aparecen representados un número mayor de grupos taxonómicos. Allí la incorporación de peces, foraminíferos y poríferos fue más frecuente (Tabla 1). La ingesta de poliquetos, en cambio, no parece estar relacionada con el sitio (Tabla 2). En términos de abundancia, copépodos, anfípodos y ostrácodos resultan más importantes en el sector central, al igual que equinoideos y holoturoideos, escasamente representados en el área norte.

El método MDS (Fig. 2) muestra el ordenamiento de las muestras en base a la similitud en la composición de la dieta. Las muestras 1, 2, 3, 4 y 8 aparecen formando un grupo; estas muestras corresponden al área norte, mientras que las muestras del centro 5, 6, 7, 9 y 10 forman un segundo grupo; esta ordenación convalida las diferencias halladas entre los sitios. 
Tabla 1

Frecuencias de ocurrencia expresadas en porcentaje, para las distintas muestras. Las muestras M1, M2, M3, M4 y M8 corresponden al sector norte. Las muestras M5, M6, M7, M9 y M10 corresponden al área central del golfo

Frequencies of occurrence in percentage for the different samples. Samples M1, M2, M3, M4 and M8 belong to the northern area. Samples M5, M6, M7, M9 and M10 belong to the central area of the gulf

\begin{tabular}{cccccccccccc}
\hline & M 1 & M 2 & M 3 & M 4 & M 8 & M 6 & M 7 & M 5 & M 9 & M10 \\
\hline MOP & 97 & 97 & 80 & 93 & 90 & 57 & 73 & 63 & 67 & 70 \\
Diatomeas & 33 & 10 & 0 & 0 & 10 & 7 & 7 & 7 & 3 & 7 \\
Foraminíferos & 0 & 10 & 7 & 7 & 0 & 13 & 13 & 17 & 10 & 23 \\
Poríferos & 0 & 0 & 3 & 0 & 7 & 10 & 3 & 0 & 7 & 7 \\
Nematodos & 43 & 33 & 37 & 17 & 30 & 23 & 20 & 30 & 33 & 17 \\
Poliquetos & 57 & 57 & 47 & 53 & 33 & 20 & 33 & 33 & 26 & 40 \\
Decápodos & 73 & 80 & 70 & 67 & 73 & 43 & 40 & 43 & 33 & 33 \\
Otros crustáceos & 17 & 13 & 17 & 10 & 20 & 13 & 7 & 10 & 13 & 13 \\
Peces & 0 & 7 & 0 & 0 & 0 & 7 & 3 & 10 & 7 & 7 \\
Indeterminados & 50 & 57 & 47 & 33 & 20 & 23 & 17 & 17 & 30 & 20 \\
\hline
\end{tabular}

Tabla 2

Abundancias relativas expresadas en porcentaje, para las distintas muestras. Las muestras M1, M2, M3, M4 y M8 corresponde al sector norte. Las muestras M5, M6, M7, M9 y M10 corresponden al área central del golfo

Relative abundances expressed in percentage for the different samples. Samples M1, M2, M3, M4 and M8 belong to the northern area and samples M5, M6, M7, M9 and M10 belong to the central area of the gulf

\begin{tabular}{|c|c|c|c|c|c|c|c|}
\hline Muestra & MOP & Poliquetos & $\begin{array}{l}\text { Crustáceos } \\
\text { decápodos }\end{array}$ & Nematodos & Protistas & Otros & Indeterminados \\
\hline M1 & 33,57 & 14,12 & 31,07 & 4,27 & 1,80 & 5,98 & 9,19 \\
\hline M2 & 31,63 & 20,40 & 20,92 & 3,12 & 2,14 & 8,02 & 13,77 \\
\hline M3 & 32,51 & 22,08 & 29,10 & 2,88 & 1,54 & 4,83 & 7,06 \\
\hline M4 & 31,08 & 11,55 & 31,76 & 1,51 & 1,48 & 10,98 & 11,64 \\
\hline M5 & 30,19 & 12,43 & 16,58 & 3,03 & 7,32 & 16,97 & 13,48 \\
\hline M6 & 33,25 & 11,67 & 14,82 & 4,29 & 9,06 & 11,56 & 15,35 \\
\hline M7 & 28,65 & 13,48 & 15,16 & 5,33 & 9,04 & 12,64 & 15,70 \\
\hline M8 & 30,31 & 13,09 & 30,63 & 4,07 & 2,52 & 7,02 & 12,36 \\
\hline M9 & 29,30 & 12,09 & 15,63 & 5,25 & 8,71 & 13,07 & 15,95 \\
\hline M10 & 28,41 & 21,22 & 14,74 & 4,67 & 7,91 & 9,75 & 13,30 \\
\hline
\end{tabular}




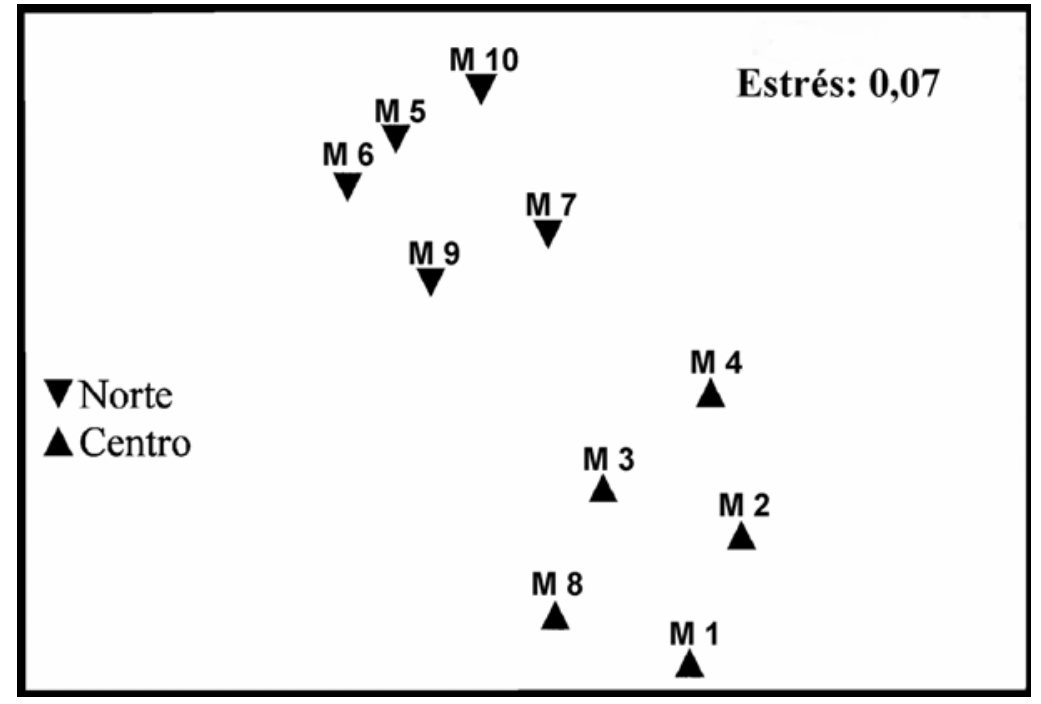

Figura 2

Ordenamiento de las muestras de Munida gregaria, por el método “multidimensional scaling” (MDS). Un estrés de 0,07 significa que la representación bidimensional es útil

Multidimensional scaling (MDS) ordination by sample of Munida gregaria.

A stress of 0.07 means that the two-dimensional representation is useful

Tabla 3

Contribución de cada uno de los componentes alimenticios a la diferenciación entre los sitios central y norte del Golfo San Jorge, Argentina

Contribution of each food component to the differentiation between northern and central areas of the San Jorge Gulf, Argentina

\begin{tabular}{lcccc}
\hline & $\begin{array}{c}\text { Abundancia } \\
\text { Norte } \\
(\%)\end{array}$ & $\begin{array}{c}\text { Abundancia } \\
\text { Centro } \\
(\%)\end{array}$ & $\begin{array}{c}\text { Contribución } \\
\text { diferencia } \\
(\%)\end{array}$ & $\begin{array}{c}\text { Contribución } \\
\text { acumulada } \\
(\%)\end{array}$ \\
\hline Decápodos & 28,70 & 15,39 & 35,22 & 35,22 \\
Protistas & 1,90 & 8,41 & 17,23 & 52,45 \\
Otros & 7,37 & 12,80 & 14,63 & 67,08 \\
Poliquetos & 16,25 & 14,18 & 11,74 & 78,82 \\
Indeterminados & 10,80 & 14,76 & 10,62 & 89,44 \\
MOP & 31,82 & 29,96 & 6,50 & 95,94 \\
\hline
\end{tabular}

Dichas diferencias se deben principalmente al efecto de los crustáceos decápodos en la dieta, los cuales son más abundantes en la alimentación en el sector norte. Otro componente importante en la diferenciación de los sitios son los protistas o restos de los mismos, compuestos principalmente por frústulos de diatomeas y foraminíferos, los cuales están mejor representados en las muestras del sector central (Tabla 3).

\section{Discusión}

En el Golfo San Jorge, el método principal de obtención de energía de la especie es la alimentación sobre los depósitos de materia orgánica superficiales y la necrofagia (Vinuesa \& Varisco no publicado). En este trabajo la materia orgánica particulada (MOP) ha sido también el componente más importante de la dieta, independientemente del sector estudiado. Pérez Flores 
(1991), al comparar las dos cámaras que conforman el estómago de Pleuroncodes planipes, indicó que la materia orgánica hallada era producto de la ingestión y no de la digestión.

La incidencia baja de las algas se debería a la ausencia de las mismas en las comunidades de fondos blandos, en las que la langostilla es característica. En profundidades menores a los $35 \mathrm{~m}$, sólo Macrocystis pyrifera aparece como abundante en áreas donde se halla presente la langostilla. Sin embargo, no se registró ninguna especie de macroalga abundante a profundidades mayores en el Golfo San Jorge (Roux et al. 1995, Vinuesa et al. 1998).

Algunos ítems pueden haber sido sobreestimados, al no determinar si realmente constituían un alimento o bien formaban parte del sedimento biogénico incorporado. Entre estos ítems encontramos foraminíferos, poríferos, equinodermos y moluscos, los cuales fueron identificados a partir de restos duros como caparazones, espículas, rádulas y fragmentos de valvas. Es posible que el método de preservación de las muestras afecte la conservación de los foraminíferos.

Los crustáceos con caparazones no muy calcificados, algunos equinodermos y algunos peces presentan daños y mortalidades frecuentes durante el descarte en pesquerías de arrastre, por lo que pasan a ser presas de aves, peces y carroñeros bentónicos (Hill \& Wassenberg 1990, Kaiser \& Spencer 1995, Philippart 1998, Bergman et al. 2001, Stobutzki et al. 2001). En el caso de la centolla (L. santolla) y langostilla (M. gregaria), se han observado elevadas mortalidades y número de lesiones en el área central del golfo (Vinuesa et al. 1998, Balzi 1999). En la pesca de langostino, los organismos más afectados son el erizo Trypilaster philippii y el poliqueto maldánido Asychis amphiglypta (Roux 2000).

Los equinodermos Pseudechinus magellanicus y T. philippii, son los invertebrados más numerosos en la captura incidental en la pesquería de langostino (Roux 2000); sin embargo, no representarían una presa apetecible, ya que los erizos mostraron una importancia menor en la dieta de la especie. Este hecho también ha sido destacado en estudios realizados en el canal Beagle (Romero et al. 2004).

Los peces son un componente importante del descarte en los distintos caladeros, principalmente la merluza Merluccius hubbsi. Se ha calculado que en
1997, la flota langostinera ha descartado aproximadamente unas $20.000 \mathrm{t}$ de esta especie (Pettovelo 1999). Otros cálculos más recientes señalan que entre las distintas especies se devuelven al agua más de $5.000 \mathrm{t}$ por año en el Golfo San Jorge (CEDEPESCA 2005) ${ }^{1}$. Es posible que su importancia en la dieta de la langostilla se halle subestimada por no ser identificable en el presente trabajo, dado que se trata de un componente blando, con tejidos fácilmente disgregables que forman MOP. También es probable, como sucede en otras pesquerías, que pocos peces lleguen al fondo, siendo alimento de numerosas especies de aves, de delfines y otros peces (Gislason 2003).

Las diferencias en la ingesta de crustáceos decápodos estarían relacionadas con el descarte de langostilla ( $M$. gregaria) y langostino ( $P$. muelleri) pequeño asociado a los lances de pesca del sector norte. La identificación de $P$. muelleri en los contenidos estomacales de la langostilla consolida la existencia de una relación directa entre la pesquería de este recurso y la alimentación de $M$. gregaria en el área norte del golfo. Por otra parte, la incorporación de individuos coespecíficos en la dieta ha sido citada en el Golfo San Jorge y otras áreas donde la especie se presenta (Zeldis 1985, Romero et al. 2004, Vinuesa \& Varisco no publicado). La ingesta de individuos coespecíficos sería el resultado de prácticas carroñeras y no de captura activa, ya que la langostilla solo estaría morfológicamente capacitada para atrapar ejemplares de tallas pequeñas y en períodos de postmuda (Romero 2003). Además, durante gran parte del año se ha comprobado que machos adultos y hembras y juveniles se hallan separados espacialmente, dado que los machos migran a zonas costeras en el periodo reproductivo y luego regresan a aguas más profundas (Vinuesa 2007).

El descarte en el golfo aparece indicado como responsable en primera instancia del aumento poblacional de algunos crustáceos y equinodermos de hábitos generalistas (Roux 2000). Estudios previos efectuados en la centolla (Lithodes santolla) mostraron una disminución en la cantidad de componentes de la dieta de esta especie, atribuible a la utilización del descarte (Balzi 1999, Vinuesa et al. 1998). Este efecto del descarte sobre la alimentación redunda en una diversidad menor de componentes identificados en las muestras del sector norte respecto del área central, donde los descartes son menos importantes.

La alimentación de la langostilla está influenciada por la oferta local de alimento. Dado que ambos sitios 
estudiados corresponden a un agrupamiento faunístico similar, característico de fondos blandos (Roux et al. 1995), las diferencias aquí halladas en la alimentación son debidas a la composición del descarte de la pesca, la cual presenta características particulares según la pesquería que se desarrolla en el área. De esta forma alimentarse de MOP y carroña resulta un método económico en términos energéticos, principalmente en sitios de acumulación de productos del descarte, permitiéndole aprovechar a la especie esa materia orgánica disponible. Si bien un análisis comparativo entre áreas libres de descartes y sitios de actividad pesquera intensa reflejaría mejor las variaciones en la dieta debidas al descarte, se pone aquí en evidencia la influencia de esta práctica en la alimentación de la especie en el Golfo San Jorge.

\section{Agradecimientos}

Se agradece la colaboración de la Mg. Susana Risso y del Lic. H. Zaixso, quien también revisó y realizó sugerencias sobre el manuscrito. A los Sres. Ezequiel Murphy y Ricardo Álvarez, de la Secretaría de Pesca de la provincia del Chubut, por la obtención de muestras. Se agradece a los evaluadores del trabajo, por las valiosas sugerencias realizadas.

\section{Literatura citada}

Balzi P. 1999. Los hábitos alimenticios de la centolla, Lithodes santolla (Molina) del Golfo San Jorge. Naturalia Patagónica, Ciencias Biológicas 5: 67-87.

Bergman M, DJ Beare \& PG Moore. 2001. Damage sustained by epibenthic invertebrates discarded in the Nephrops fishery of the Clyde Sea area. Journal of Sea Research 45: 105-118.

Bertolotti MI, GA Verazay, E Errazti, AN Pagani \& JJ Bosco. 2001. Flota pesquera Argentina. Evolución durante el periodo 1960-1998, con una actualización al 2000. En: Bertolotti MI, GA Verazay \& R Akselman (eds). El Mar Argentino y sus recursos pesqueros, pp. 953. Instituto Nacional de Investigación y Desarrollo Pesquero, Mar del Plata.

Boschi EE, MI Iorio \& C Fischbach. 1992. Catálogo ilustrado de los crustáceos estomatópodos y decápodos marinos de Argentina. Frente Marítimo 10: 7-94.

Clarke KR \& RM Warwick. 2001. Change in marine communities: An approach to statistical analysis and interpretation, 173 pp. $2^{\text {nd }}$ Edition. Primer-E Ltd., Plymouth.

Davis MW. 2002. Key principles for understanding fish bycatch discard mortality. Canadian Journal of Fisheries and Aquatic Sciences 59: 1834-1843.

Gislason H. 2003. The effects of fishing on non-target species and ecosystem structure and function. En: Sinclair M \& G Valdimarsson (eds). Responsible fisheries in the marine ecosystem, pp. 255-274. FAO, Roma.

Goñi R. 1998. Ecosystem effects of marine fisheries: An overview. Ocean and Coastal Management 40: 37-64.

Hill BJ \& TJ Wassenberg. 1990. Fate of discards from prawn trawlers in Torres Strait. Australian Journal of Marine and Freshwater Research 41: 53-64.

Kaiser MJ \& BE Spencer. 1995. Survival of by-catch from a beam trawl. Marine Ecology Progress Series 126: 31-38.

Lovrich G, M Casalinuovo, S Molina, C Cárcamo \& R Pierotti. 1998. Las langostillas Munida subrugosa y $M$. gregaria (Decapoda, Anomura) como potencial recurso económico patagónico. Naturalia Patagónica, Ciencias Biológicas 6: 89-92.

Penchaszadeh P, G Bigatti \& P Miloslavich. 2004. Feeding of Pseuchinus magellanicus (Philippi, 1857) (Echinoidea: Temnopleuridae) in the SW Atlantic coast (Argentina). Ophelia 58 (2): 91-99.

Pérez Barros P, ME D’Amato \& GA Lovrich. 2005. Munida gregaria vs. Munida subrugosa (Decapoda: Anomura): the beginning of the end of two different species? Berichte zur Polar-und Meeresforschung 507: $172-174$

Pérez Barros P, ME D’Amato \& GA Lovrich. En prensa. Taxonomic status of two South American sympatric squat lobsters, Munida gregaria and M. subrugosa. Proceedings of the Biological Journal of the Linnean Society.

Pérez Flores F. 1991. Alimentación de la langostilla Pleuroncodes planipes (Stimpson, 1860), durante el periodo de reproducción en la costa occidental de Baja California Sur. Tesis de Licenciatura, Universidad Nacional Autónoma de México, 70 pp.

Pérez Flores R \& D Aurioles Gamboa. 1995. Hábitos alimentarios de la langostilla bentónica en la plataforma continental de la costa oeste de Baja California sur. En: Aureoles-Gamboa D \& EF Balart (eds). La langostilla: Biología, Ecología y Aprovechamiento, pp. 125-137. Centro de Investigaciones Biológicas del Noroeste. La Paz, Baja California. 
Pettovelo AD. 1999. By-catch in the Patagonian red shrimp (Pleoticus muelleri) fishery. Marine and Freshwater Research 50: 123-127.

Philippart CJ. 1998. Long term impact of bottom fisheries on several by-catch species of demersal fish and benthic invertebrates in the south-eastern North Sea. ICES Journal of Marine Science 55: 342-352.

Rodríguez L \& $\mathbf{R}$ Bahamonde. 1986. Contribución al conocimiento de Munida subrugosa (White, 1847) en la XII Región, Chile. En: Arana P (ed). La Pesca en Chile, pp. 283-296. Universidad Católica de Valparaíso, Valparaíso.

Romero M. 2003. Rol trófico de la langostilla Munida subrugosa (Crustacea: Decapoda) del Canal de Beagle, Tierra del Fuego, Argentina. Tesis Doctoral, Universidad Nacional de Córdoba, 215 pp.

Romero M, G Lovrich, F Tapella \& S Thatje. 2004. Feeding ecology of the crab Munida subrugosa (Decapoda: Anomura: Galatheidae) in the Beagle Channel, Argentina. Journal of the Marine Biological Association of the United Kingdom 84: 359-365.

Roux A. 2000. Evaluación del impacto pesquero a través del análisis de la fauna bentónica acompañante en la pesquería de langostino (Pleoticus muelleri) del Golfo San Jorge y litoral de Chubut, Argentina. Frente Marítimo 18: $143-149$

Roux A \& M Fernández. 1997. Caracterización de los fondos de pesca de langostino patagónico (Pleoticus muelleri) en el Golfo San Jorge y litoral de la provincia de Chubut - Argentina. Informe Técnico INIDEP 13: 127.

Roux A, M Fernández \& C Bremec. 1995. Estudio preliminar de las comunidades bentónicas de los fondos de pesca del Golfo San Jorge (Argentina). Ciencias Marinas 21: 295-310.
Stobutzki I, M Millar \& D Brewer. 2001. Sustainability of fishery bycatch: a process for assessing highly diverse and numerous bycatch. Environmental Conservation 28: $167-181$

Tapella F. 2002. Reproducción, crecimiento, distribución y abundancia de la langostilla Munida subrugosa (Anomura: Galatheidae) del canal Beagle, Tierra del Fuego, Argentina. Tesis Doctoral, Universidad Nacional de Córdoba, 147 pp.

Tapella F, GA Lovrich, MC Romero \& S Thatje. 2002. Reproductive biology of the crab Munida subrugosa (Decapoda: Anomura: Galatheidae) in the Beagle Channel, Argentina. Journal of the Marine Biological Association of the United Kingdom 82: 589-595.

Vinuesa JH. 2005. Distribución de crustáceos decápodos y estomatópodos del Golfo San Jorge. Revista de Biología Marina y Oceanografía 40(1): 7-21.

Vinuesa JH. 2007. The reproduction of Munida gregaria (Decapoda, Galatheidae) in San Jorge Gulf, South-West Atlantic Ocean. Journal of Crustacean Biology 27: 437444.

Vinuesa JH, P Balzi \& GA Lovrich. 1998. La centolla (Lithodes santolla) del Golfo San Jorge. Contribuciones Científicas CADIC, Ushuaia 32: 1-32.

Williams BG. 1973. The effect of the environment on the morphology of Munida gregaria (Fabricius). Crustaceana 24: $197-210$

Williams BG. 1980. The pelagic and benthic phases of postmetamorphic Munida gregaria (Fabricius) (Decapoda, Anomura). Journal of Experimental Marine Biology and Ecology 42: 125-141.

Zeldis J. 1985. Ecology of Munida gregaria (Decapoda, Anomura): distribution and abundance, population dynamics and fisheries. Marine Ecology Progress Series 22: 77-99. 